\title{
BMJ Reductions in hospital admissions and open mortality rates observed after integrating emergency care: a natural experiment
}

\author{
Adrian A Boyle, ${ }^{1}$ Vazeer Ahmed, ${ }^{1}$ Christopher R Palmer, ${ }^{2}$ Tom J H Bennett, ${ }^{3}$ \\ Susan M Robinson ${ }^{1}$
}

To cite: Boyle AA, Ahmed V, Palmer CR, et al. Reductions in hospital admissions and mortality rates observed after integrating emergency care: a natural experiment. BMJ Open 2012;2:e000930.

doi:10.1136/

bmjopen-2012-000930

- Prepublication history for this paper is available online. To view this file please visit the journal online (http://dx. doi.org/10.1136/ bmjopen-2012-000930).

Received 24 January 2012 Accepted 8 June 2012

This final article is available for use under the terms of the Creative Commons Attribution Non-Commercial 2.0 Licence; see http://bmjopen.bmj.com

${ }^{1}$ Emergency Department, Cambridge University Foundation Hospitals NHS Trust, Cambridge, UK ${ }^{2}$ Centre for Medical Statistics, Cambridge University, Cambridge, UK ${ }^{3}$ Cambridge University Foundation Hospitals Trust, Cambridge, UK

Correspondence to Dr Adrian A Boyle; adrian. boyle@addenbrookes.nhs.uk

\section{ABSTRACT}

Objectives: Reducing emergency admissions is a priority for the NHS. A single hospital's emergency care system was reorganised with the principles of front-loaded investigations, integration of specialties, reduced duplication, earlier decision making by senior clinicians and a combined emergency assessment area. The authors relocated our Medical Assessment Unit into our emergency department in 2006. The authors evaluated changes in admissions and mortality before and after 2006, compared with other similar hospitals.

Design: Quasi-experimental before and after study using routinely collected data.

Setting and participants: 1 acute hospital in England, the intervention site, was compared with 23 other English hospitals between 2001 and 2009.

Outcome measures: Our outcome measures were hospital standardised mortality ratios (HSMRs) for non-elective admissions and standardised admission ratios (SARs).

Results: The authors observed a statistically and clinically significant decrease in HSMR and SAR. The intervention hospital had the lowest HSMR and SAR of all the hospitals in our sample. This was statistically significant, $p=0.0149$ and $p=0.0002$, respectively.

Conclusion: Integrating emergency care in one location is associated with a meaningful reduction in mortality and emergency admissions to hospital.

\section{INTRODUCTION}

There have been large increases in the number of emergency department attendances and emergency admissions over the past 10 years, policy initiatives have largely been ineffective at reducing this. ${ }^{1}$ Emergency admissions now cost the NHS $£ 11$ billon a year. ${ }^{2}{ }^{3}$ In addition, emergency admissions disrupt elective care. ${ }^{1}$ Secondary emergency care systems have evolved rather than developed by any planned process. While it has always been recognised that a part of an acute hospital needs to accessible for unplanned emergencies, there is less consensus on the

\section{ARTICLE SUMMARY}

\section{Article focus}

- We describe the improvements that occur when a medical admissions unit is closed and relocated into an emergency department. We compared a single unit to a family of similar hospitals. We used routine data analysed by the Dr Foster healthcare intelligence unit.

\section{Key messages}

- Reducing duplicate processes, centralising hospital emergency care in one area and streamlining non-elective admission pathways is associated with sustained reductions in non-elective admissions and mortality.

- The Standardised Admissions Ratio is an effective way to measure the performance of nonelective admissions.

Strengths and limitations of this study

- This study has compared performance against controls over a ten year period.

- The study uses retrospectively analysed administrative data.

optimal organisation of unplanned emergency care. Emergency care systems vary widely across the world and even within countries and between hospitals in different countries. An emergency department can be seen as having an advanced triage role, identifying and treating ill and injured while discharging safely the well. An ideal, publicly funded, hospital emergency care system would save lives, reduce morbidity while minimising admissions to hospital. The degree of investigation and management before admission varies widely. Medical Admissions Units (MAUs), where general practitioners (GP) can refer patients directly to a general physician, are common throughout the UK. Surgical Admission Units, where general practitioners can refer patients directly to a surgeon are also common. Patients referred to these admission units usually bypass emergency 
departments. These admission units also receive referrals from the hospital emergency department. In the UK, around $50 \%$ of all emergency admissions to hospital pass through the emergency department.

While this model of care is well understood by healthcare staff, it results in long delays for definitive treatment and fragmented care. Patient may undergo multiple assessments if they pass through an emergency department and are then subsequently admitted to an assessment unit. Many bed moves result, which compromises infection control and continuity of care. In 2004, we embarked on a service redesign of the emergency assessment and admission process. We mapped out admission pathways for patients who were admitted non-electively. We sought to remove wasteful steps and duplication. It was apparent that the process of admitting general medical patients (the majority of nonelective admissions) to our hospital was fragmented, inequitable, complex and had multiple, duplicated steps. We found that patients often passed through two assessment areas, had documentation on two separate medical records, had blood tests repeated and might be assessed by up to five different doctors.

We opened the Emergency Assessment Unit (EAU) in 2006. The two diagrams show the process for admitting or discharging the majority of emergency patients presenting to our hospital. This was broadly similar to admission process in most acute hospitals in the UK before 2006 and radically different afterwards. We redesigned the service, so that the majority of non-elective admissions attended the emergency department. In 2006, we closed our MAU and relocated the staff to the emergency department. We expanded the emergency department by about the number of beds that the MAU had had. We developed a combined clerking process that was supported by shared documentation, with the overall aim of reducing assessments (figures 1 and 2). The emergency department was supported by the development of the short stay medical and surgical wards, a clinical decisions unit and a children's observation unit. These wards aimed to look after patients for no more than 3 days. There was an increased access to emergency radiology. The proportion of non-elective admissions entering the hospital through the emergency department rose from about $50 \%$ to around $80 \%$. Patients requiring admission from the outpatient department, referrals from other hospitals and obstetric patients continued to bypass the emergency department. We did not use an explicit theoretical or scientific framework to guide us, but our approach had elements of lean manufacturing techniques in that we performed value stream mapping of patient pathways. ${ }^{4}$ Our approach differed from 'lean' in that there was a 'big bang launch', and there was less emphasis on continuous improvement in this program than a 'lean model'.

Previous small-scale evaluations identified that this model of care was associated with a reduction in admissions and hospital standardised mortality. ${ }^{5}{ }^{6}$ However, these studies were compromised by short follow-up times and an absence of meaningful controls. We aimed to evaluate whether our model of care was associated with reduced admissions and inpatient mortality, compared with other similar hospitals. Our outcome measures were hospital standardised mortality ratios (HSMRs) for nonelective admissions and standardised admission ratios (SARs). We anticipated, based on previous work, that our admissions ratios would be lower, but we aimed to evaluate against similar hospitals.

\section{METHODS}

Addenbrookes is a teaching hospital in Cambridge, England. The emergency department sees around 90000 patients per annum. We compared this hospital's performance with a family of most similar hospitals trusts. These were mainly teaching hospitals trusts outside London. This family of hospitals are frequently compared for operational performance. These 15 acute trusts are identified in box 1 , comprising 23 emergency departments.

We conducted a retrospective evaluation using Dr Foster's data. This is a healthcare intelligence unit that
Figure 1 Traditional process for evaluating patients presenting for unscheduled care before 2006. ED, Emergency Department; MAU, Medical Admissions Unit.

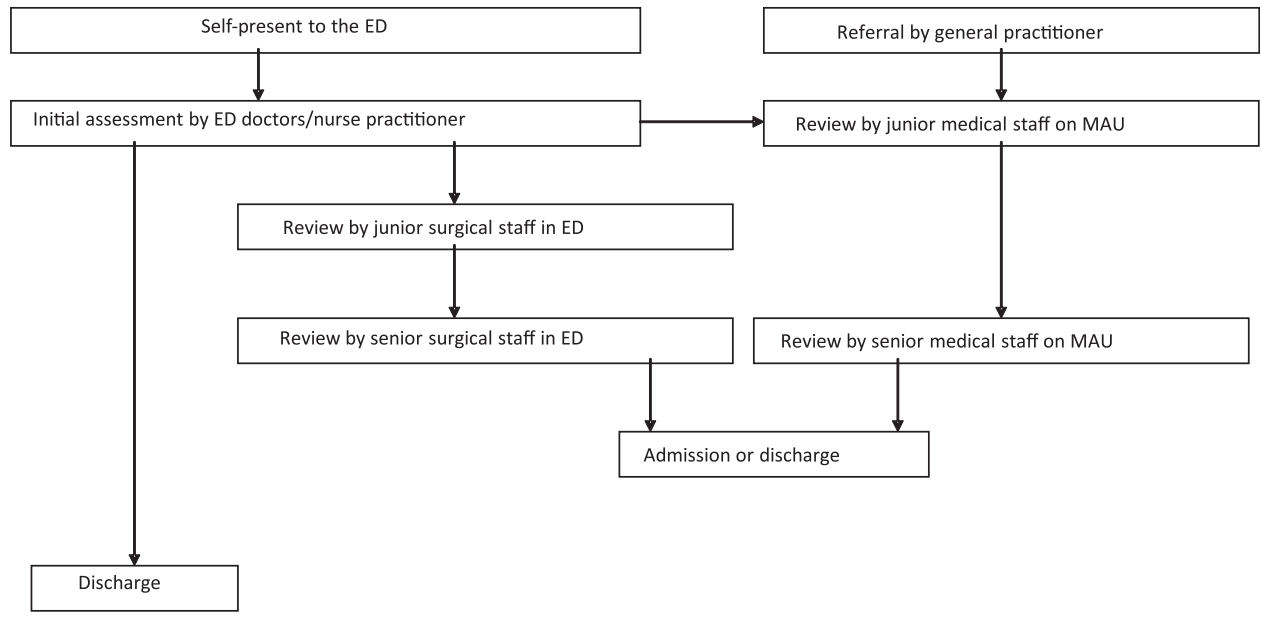




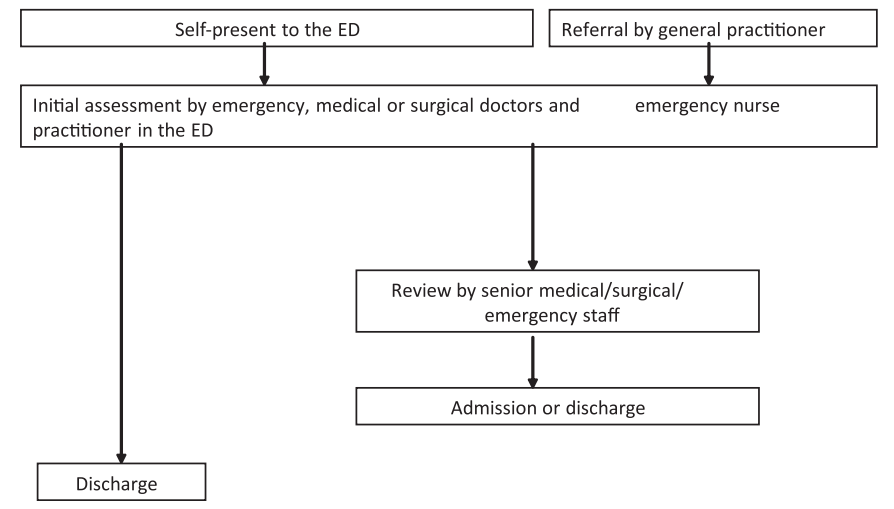

Figure 2 Streamlined process for evaluating patients presenting for unscheduled care from 2006.

analyses data from all the hospitals in the NHS. We evaluated HSMR for non-elective admissions over the years immediately before and after implementation of our integrated emergency care model in 2006. HSMR is a performance measure used widely throughout the NHS and has high face validity. HSMR is the ratio of the observed number of inhospital deaths during admissions with an HSMR diagnosis to the expected number of deaths, multiplied by 100 . The expected number of deaths is actuarially based on a number of conditions with a predictable rate of death, including heart failure, strokes and fractured neck of femur. We ran the model with and without adjusting for socioeconomic deprivation. This made no practical difference to our results or conclusions, so we have reported the results without adjusting for socioeconomic deprivation. HSMR is reported throughout the NHS. HSMR is calculated for non-elective admissions regardless of whether they are admitted through an assessment unit or emergency department. We benchmarked our performance against

Box 1 Family of similar hospitals

Cambridge University Hospitals NHS Foundation Trust Central Manchester University Hospitals NHS Foundation Trust

Derby Hospitals NHS Foundation Trust

Lancashire Teaching Hospitals NHS Foundation Trust

Leeds Teaching Hospitals NHS Trust

Nottingham University Hospitals NHS Trust

Oxford Radcliffe Hospitals NHS Trust

Royal Liverpool and Broadgreen University Hospitals NHS

Trust

Salford Royal NHS Foundation Trust

Sheffield Teaching Hospitals NHS Foundation Trust

Southampton University Hospitals NHS Trust

The Newcastle Upon Tyne Hospitals NHS Foundation Trust

University Hospital Birmingham NHS Foundation Trust

University Hospital of South Manchester NHS Foundation

Trust

University Hospitals Bristol NHS Foundation Trust

University Hospitals of Leicester NHS Trust the relevant financial year. We have previously developed a measure called the SAR with the Dr Foster unit. ${ }^{7}$ The numerator is the number of non-elective admissions. The denominator was the total number of patients registered in GP practices that refer $80 \%$ or more of their patients to an included hospital. This was then compared with a national average to provide a ratio. We benchmarked our SAR against the relevant year. We analysed data from 2001 to 2009. We conducted the analysis in the summer of 2011, as this allowed enough time to ensure that all the hospitals had provided complete returns and that all long stay patients had been discharged.

We contacted emergency medicine consultants working in all the other hospitals to establish whether their non-elective admissions to medicine or surgery were admitted through their emergency departments.

We used the method described by Palmer ${ }^{8}$ to perform hypothesis testing on the rank performance of our hospital from 2006 onwards. A statistical analysis specifically designed for post hoc assessment of routinely collected data was applied to assess the plausibility of chance for accounting for observed differences in hospital care emergency care system performance measures after the policy change was implemented. In this natural experimental setting, two key rates were separately analysed to assess performance. We did not seek ethical approval as this was a service evaluation using routinely collected anonymous data.

\section{RESULTS}

We received complete data from all the hospital trusts that we contacted for information about the organisation of their emergency care services. The Dr Foster unit provided us with complete data sets on all the acute hospital trusts that we had identified as most similar to us. No hospital other than Addenbrookes routinely received GP-referred medical patients in their emergency department during the study period. Three emergency departments, in addition to Addenbrookes, routinely received GP-referred surgical admissions.

The SAR decreased suddenly in 2006 when the EAU opened and was the lowest of all the hospitals in the sample in 2006, 2007, 2008 and 2009 (table 2). The probability one prespecified hospital out of 16 performs consistently best across all 4 years is highly statistically significant, with $\mathrm{p}=0.0002$ (figure 3 ).

There was a steady decrease in our HSMR, compared with controls during the study period (table 1). This decrease began in 2003. This was maintained and was the lowest of all the hospitals in the study in 2007, 2008 and 2009. The probability that the intervention hospital performed best out of 16 hospital trusts 3 out of 4 years is statistically significant, $\mathrm{p}=0.0149$ (figure 4). We adjusted both SAR and HSMR for deprivation but found that the effect of this was minor and did not change our conclusions. The three hospitals that directed GPreferred surgical patients to the emergency department 
Figure 3 Standardised admissions ratio: Emergency Assessment Unit model versus similar hospitals.

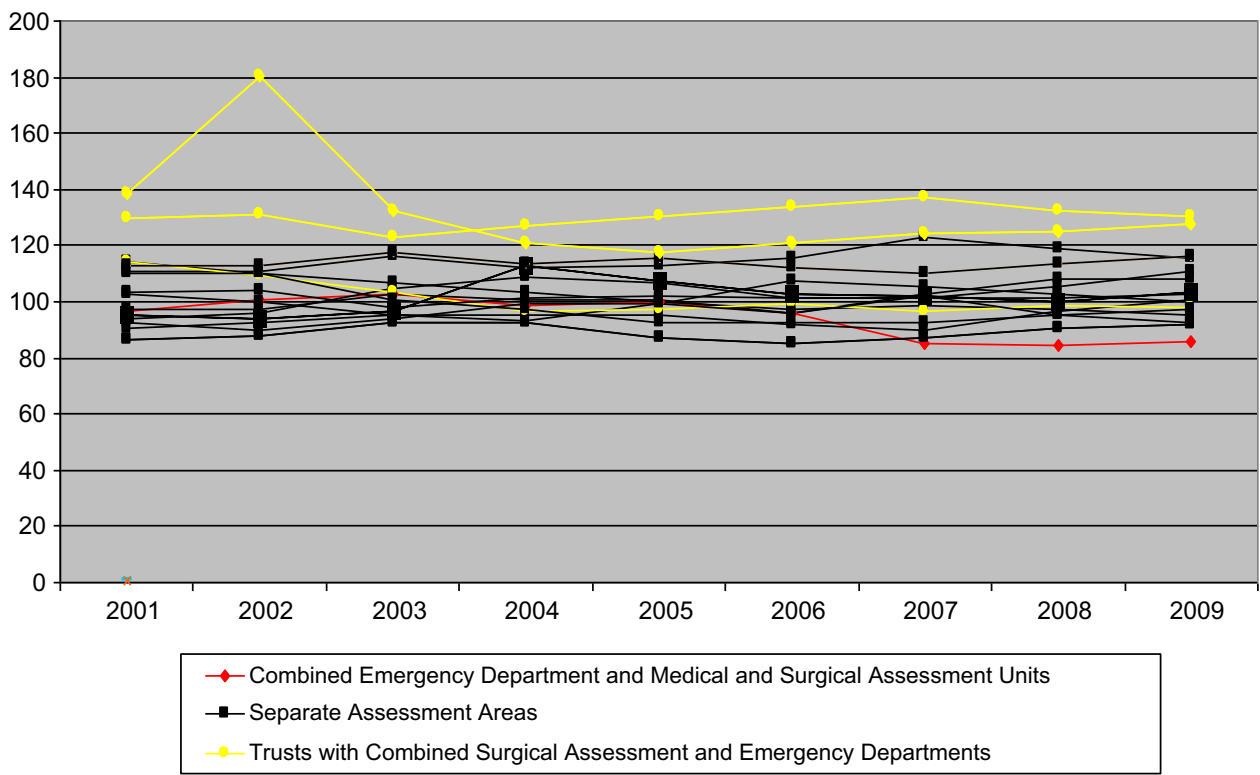

had similar performance to the other hospitals that had Surgical Admission Units.

\section{DISCUSSION}

We have found that the combining an MAU in the emergency department has been associated with a beneficial and sustained decrease in HSMR and SAR. We found that the HSMR started decreasing 2 years before the EAU opened. We found that GP-referred surgical admission units being co-located in emergency departments had little effect on HSMR or the numbers of admissions. This is not surprising as the numbers of nonelective surgical admissions and subsequent deaths are considerably lower than in patients admitted under general medical specialties.

We propose that this model of integrated emergency care allows an emergency department to fulfil its primary role of an advanced triage facility better, in that admissions are minimised safely and mortality declines. We speculate that there several reasons for reduced nonelective admissions. Many GP-referred low acuity conditions, such as suspected venous thromboembolism were attending an MAU and were counted as an admission. These patients are now rarely admitted. We think that this benefits these low acuity patients as they are usually discharged within $4 \mathrm{~h}$. We also think that this benefits the health economy, as these patients are treated more cheaply. Access to advanced imaging, such as ultrasound and CT, improved as part of this model and this allows earlier discharge.

There are some important limitations to our work. The data were routinely collected and subject to significant variability. HSMR only presents data about patients who have been admitted to hospital, not those attending
Figure 4 HSMR for non-elective admissions: Emergency Assessment Unit model compared to other similar hospitals.

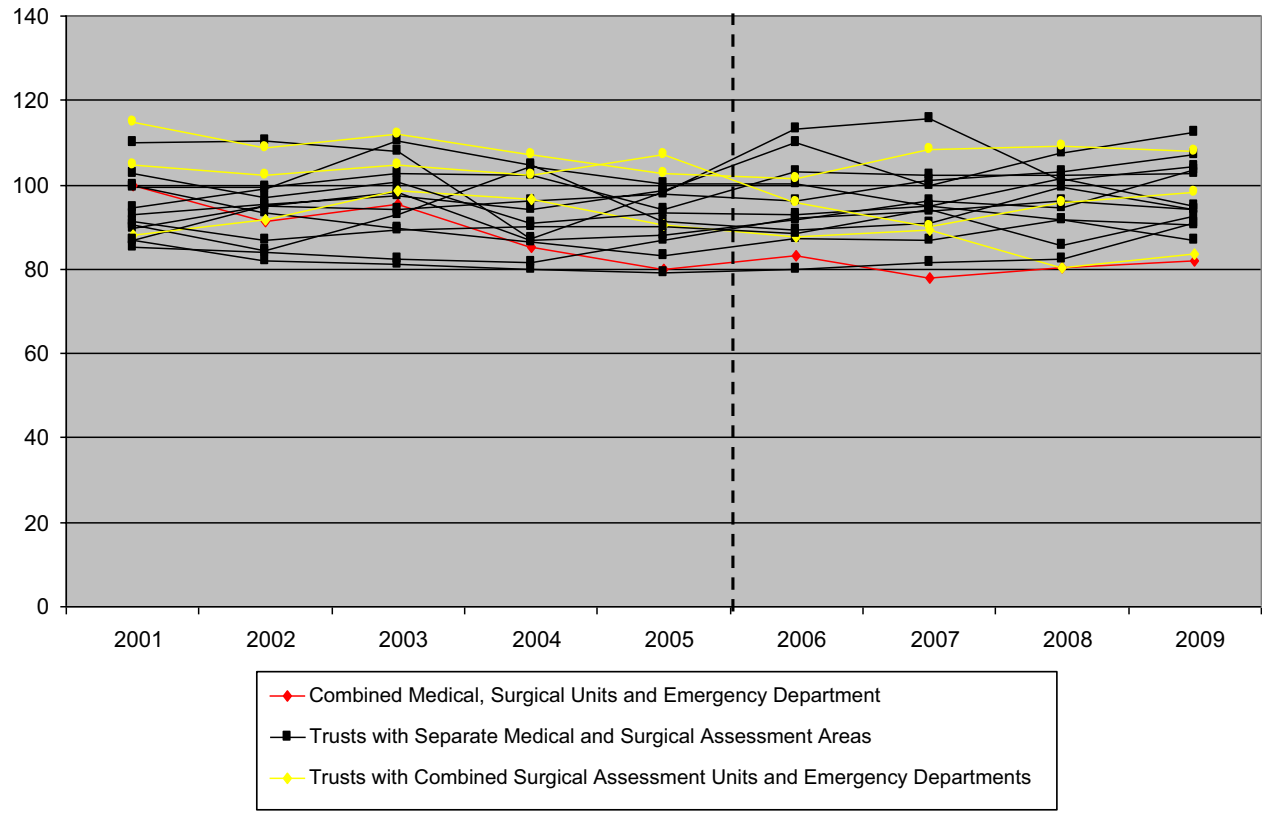




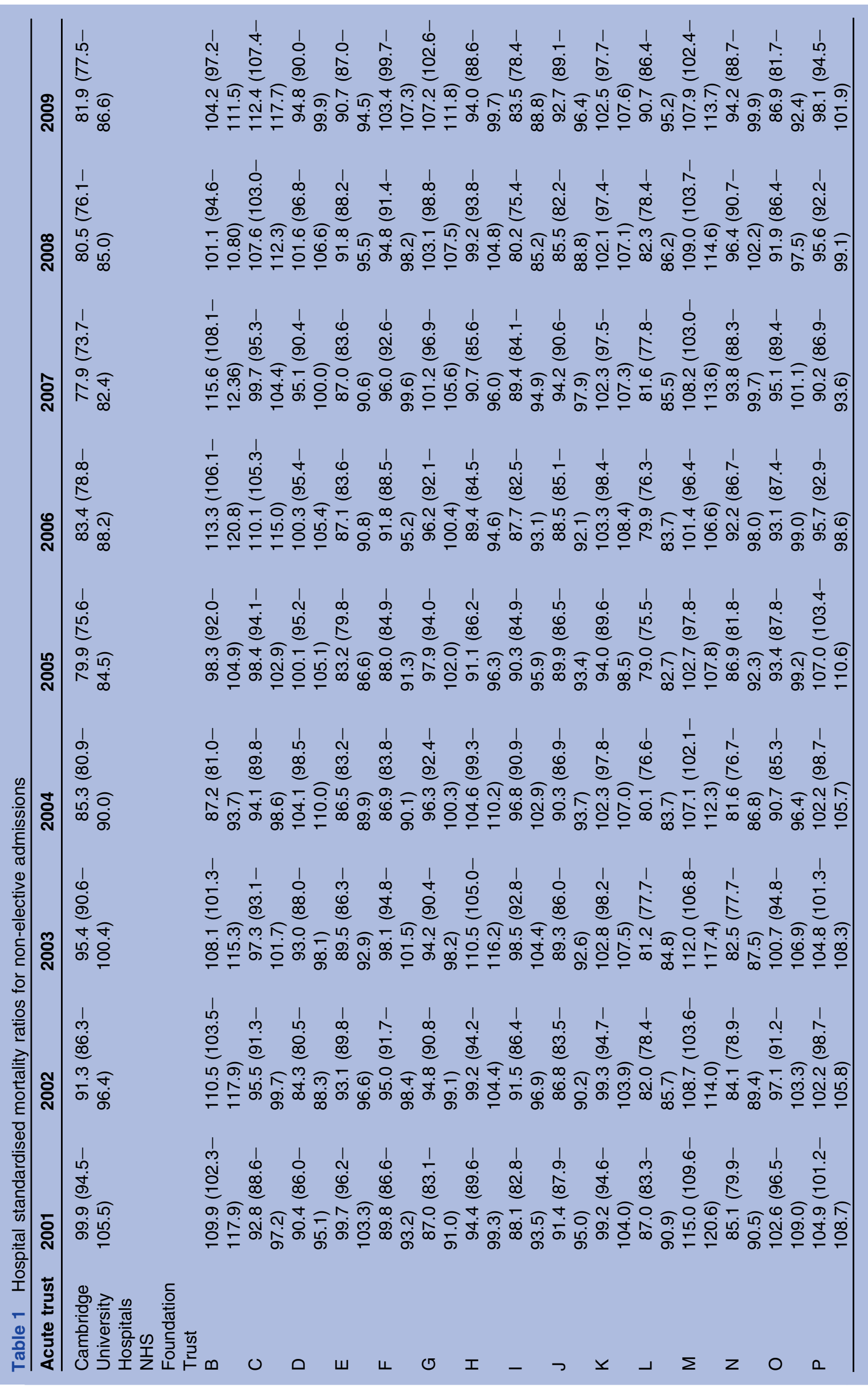




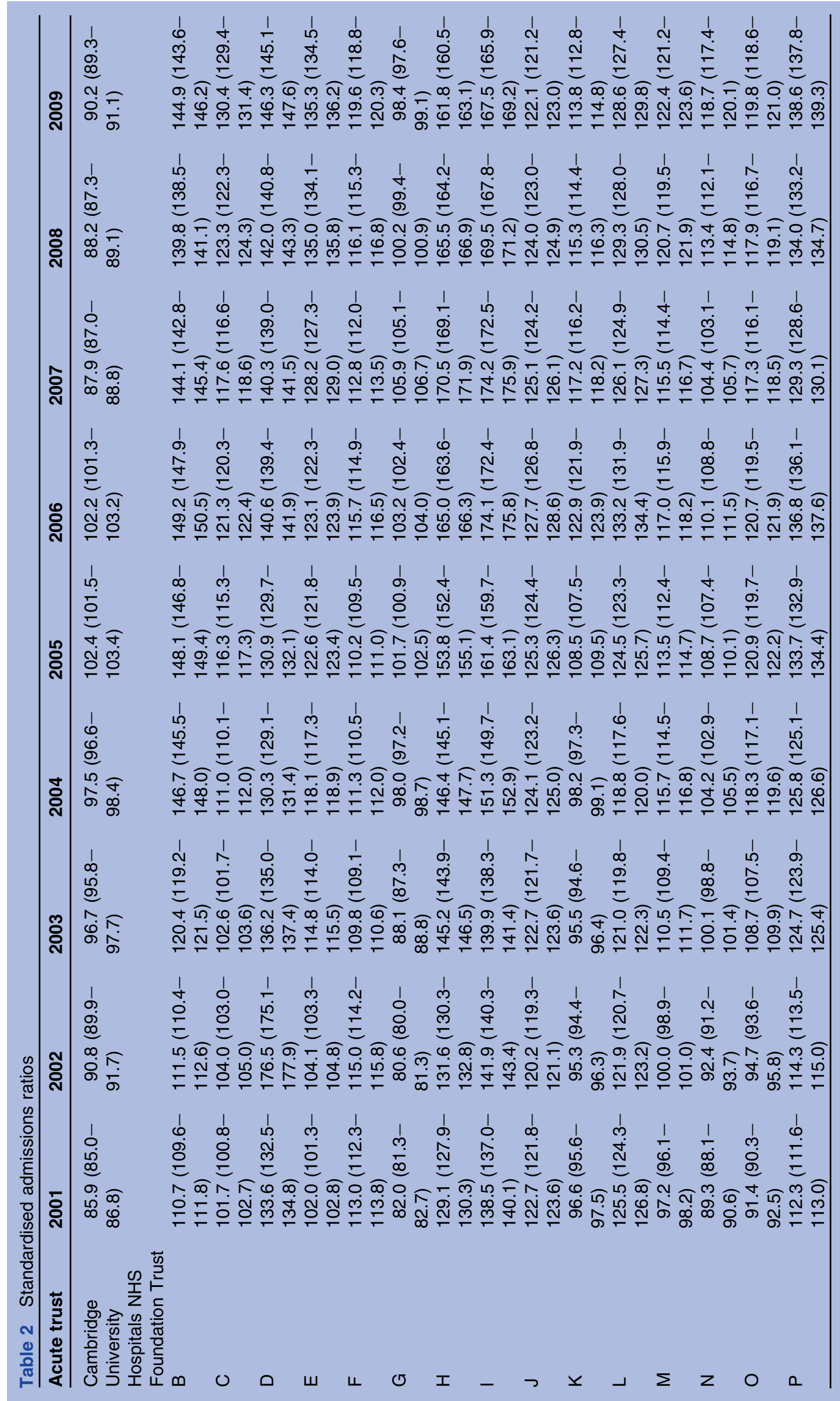


the emergency department. It is possible that our decline in HSMR might be offset by more people dying in the emergency department or dying at home. We think that this is unlikely. HSMR is an imperfect measure to assess quality of care in hospitals. ${ }^{9}{ }^{10}$ However, the use of HSMR to identify comparative trends, as we have, is less controversial. Acute hospitals in the England are obliged to report these data for performance management and follow a standard reporting system.

It is possible that the changes we saw in SAR may be due to changes in the way primary care is organised locally. We think that this is unlikely; first, we are unaware of any major changes in the way that GPs refer patients to the hospital, second, there was a big decrease just after the EAU opened, consistent with a causal relationship. We cannot ascribe a causal relationship to the changes that we have seen with our study design, a natural experiment design only allows description of associations.

The measure of admissions, SARs, can be criticised in that we had to develop a new measure. However, other performance measures available are designed for emergency departments and not the whole emergency care pathway. This measure of admissions is superior to other measures, such as conversion rate, as it provides information about a whole acute care system performance, rather than an individual department.

The $4 \mathrm{~h}$ target was introduced during the study period. This is a performance measure that mandates that $95 \%$ of patients need to have left the emergency department within $4 \mathrm{~h}$ of arrival, with financial penalties for noncompliance. This could be a potential confounding variable; however, it was applied equally to all the study hospitals.

This work comes from a single centre and care should be taken in generalising the results to other hospitals; however, the principles of reduced fragmentation and duplication of care that we have described are intuitively plausible. We have described a complex intervention, and it is difficult to be sure what parts of the intervention have lead to a beneficial response. Our study design, a natural experiment, does not allow us to state that our model causes the reduced admissions and mortality. However, combining care from multiple specialty teams, front-loading investigations, improving access to investigations and reducing duplication reduces admissions and mortality is plausible and seems to have a temporal, specific and strong effect. We did not use an explicit theoretical framework or scientific method to develop our model of care. Our approach had elements of lean manufacturing principles, but we had to be pragmatic.

We did have not presented an economic analysis of these data, this an area of future work. However, this model of care provides benefits to the whole health economy.

There is little literature that we could find about system redesign to reduce emergency hospital admissions. Most interventions are condition or presentation specific, such as falls in the elderly or frequent attenders. ${ }^{11112}$ Other studies assess the effect of general practitioners working in, or close, to the emergency department or walk-in centres. ${ }^{13-15}$ These have reported inconsistent or minor effects, at best. There is some evidence that early assessment by experienced doctors and nurses in an emergency department reduces emergency admissions. ${ }^{16-18}$ There is a large literature that examines how to improve processes within an emergency department, but little of this measures patientorientated outcomes. ${ }^{19-24}$ Future work should attempt to validate these findings across different hospitals.

\section{CONCLUSIONS}

Combining emergency admissions within one place is associated with significant and beneficial reductions in mortality and admissions. This model of integrated emergency care may allow emergency departments to fulfil their advanced triage function better.

Contributors $A A B$ and SMR initiated the project and developed the study protocol. VA and TJHB provided clinical and operational context, respectively, into the discussion. CP performed the statistical analysis. All authors contributed to the writing of the manuscript. $A A B$ and SMR conceived the project. $A A B$ and VA wrote the initial draft and analysed the results. TJHB contributed to the design and discussion. CP provided statistical analysis. All authors read and approved the final version of the paper.

Funding The study was funded by Addenbrookes Emergency Department Research Fund

\section{Competing interests None.}

Provenance and peer review Not commissioned; externally peer reviewed

Data sharing statement There are no relevant unpublished data.

\section{REFERENCES}

1. Purdy S. Avoiding Hospital Admissions. What Does the Research Evidence Say? London, UK: Kings Fund, 2010.

2. Blunt I, Bardsley M, Dixon J. Trends in Emergency Admissions in England 2004-2009: Is Greater Efficiency Breeding Inefficiency? London, UK: Nuffield Trust, 2010.

3. Stephen G. Rising hospital admissions. BMJ 2010;340:636.

4. Holweg M. The genealogy of lean production. J Oper Manag 2007;25:420-37.

5. Boyle A, Fuld J, Ahmed V, et al. Does integrated emergency care reduce mortality and non-elective admissions? A retrospective analysis. Emerg Med J 2012;29:208-12.

6. Boyle AA, Robinson SM, Whitwell D, et al. Integrated hospital emergency care improves efficiency. Emerg Med $J$ 2008;25:78-82.

7. Boyle A, Fuld J, Ahmed V, et al. Does integrated emergency care reduce mortality and non-elective admissions? A retrospective analysis. Emerg Med J 2012;29:208-12.

8. Palmer CR. Probability of recurrence of extreme data: an aid to decision-making. Lancet 1993;342:845-7.

9. Mohammed AM, Jonathan JD, Alan G, et al. Evidence of methodological bias in hospital standardised mortality ratios: retrospective database study of English hospitals. BMJ 2009;338: b780.

10. Alex B, Brian J, Paul A. Strengths and weaknesses of hospital standardised mortality ratios. BMJ 2011;342:c7116.

11. Snooks H, Cheung WY, Close J, et al. Support and Assessment for Fall Emergency Referrals (SAFER 1) trial protocol. Computerised onscene decision support for emergency ambulance staff to assess and plan care for older people who have fallen: evaluation of costs and benefits using a pragmatic cluster randomised trial. BMC Emerg Med 2010;10:2.

12. Lynch RM, Greaves I. Regular attenders to the accident and emergency department. J Accid Emerg Med 2000;17:351-4. 
13. Dale J, Lang $\mathrm{H}$, Roberts JA, et al. Cost effectiveness of treating primary care patients in accident and emergency: a comparison between general practitioners, senior house officers, and registrars. BMJ 1996;312:1340-4.

14. Salisbury C, Hollinghurst S, Montgomery A, et al. The impact of colocated NHS walk-in centres on emergency departments. Emerg Med J 2007;24:265-9.

15. Jimenez S, de la RG, Miro O, et al. [Effect of the incorporation of a general practitioner on emergency department effectiveness] (In Spanish). Med Clin (Barc) 2005;125:132-7.

16. Gonnah R, Hegazi MO, Hmdy I, et al. Can a change in policy reduce emergency hospital admissions? Effect of admission avoidance team, guideline implementation and maximising the observation unit. Emerg Med J 2008;25:575-8.

17. Thornton V, Hazell W. Junior doctor strike model of care: reduced access block and predominant Fellow of the Australasian College for Emergency Medicine staffing improve emergency department performance. Emerg Med Australas 2008;20:425-30.
18. Sen A, Hill D, Menon D, et al. The impact of consultant delivered service in emergency medicine: the Wrexham Model. Emerg Med $J$ 2012;29:366-71.

19. Dickson EW, Singh S, Cheung DS, et al. Application of lean manufacturing techniques in the Emergency Department. J Emerg Med 2009;37:177-82.

20. Holden RJ. Lean thinking in emergency departments: a critical review. Ann Emerg Med 2011;57:265-78.

21. Moskop JC, Sklar DP, Geiderman JM, et al. Emergency department crowding, part 2-barriers to reform and strategies to overcome them. Ann Emerg Med 2009;53:612-17.

22. Cooke MW, Wilson S, Pearson S. The effect of a separate stream for minor injuries on accident and emergency department waiting times. Emerg Med J 2002;19:28-30.

23. Castille K, Cooke M. One size does not fit all. View 2. Emerg Med J 2003;20:120-2.

24. Cooke MW, Higgins J, Kidd P. Use of emergency observation and assessment wards: a systematic literature review. Emerg Med $J$ 2003;20:138-42. 